\title{
Article \\ On the Weak Solutions of a Delay Composite Functional Integral Equation of Volterra-Stieltjes Type in Reflexive Banach Space
}

\author{
Ahmed M. A. El-Sayed ${ }^{1}$ (D) and Yasmin M. Y. Omar ${ }^{2, *}$ \\ 1 Faculty of Science, Alexandria University, Alexandria 21544, Egypt; amasayed@alexu.edu.eg \\ 2 Faculty of Science, Omar Al-Mukhtar University, Al-Bayda 991, Libya \\ * Correspondence: yasmin202020mohamed@gmail.com
}

check for

updates

Citation: El-Sayed, A.M.A.; Omar, Y.M.Y. On the Weak Solutions of a Delay Composite Functional Integral Equation of Volterra-Stieltjes Type in Reflexive Banach Space. Mathematics 2022, 10, 245. https://doi.org/

$10.3390 /$ math10020245

Academic Editor: Simeon Reich

Received: 7 December 2021

Accepted: 11 January 2022

Published: 13 January 2022

Publisher's Note: MDPI stays neutral with regard to jurisdictional claims in published maps and institutional affiliations.

Copyright: (c) 2022 by the authors. Licensee MDPI, Basel, Switzerland. This article is an open access article distributed under the terms and conditions of the Creative Commons Attribution (CC BY) license (https:// creativecommons.org/licenses/by/ $4.0 /)$.

\begin{abstract}
Differential and integral equations in reflexive Banach spaces have gained great attention and hve been investigated in many studies and monographs. Inspired by those, we study the existence of the solution to a delay functional integral equation of Volterra-Stieltjes type and its corresponding delay-functional integro-differential equation in reflexive Banach space $E$. Sufficient conditions for the uniqueness of the solutions are given. The continuous dependence of the solutions on the delay function, the initial data, and some others parameters are proved.
\end{abstract}

Keywords: weakly continuous; Volterra-Stieltjes integral; reflexive Banach space; continuous dependence

\section{Introduction}

The existence of weak solutions for ordinary differential equations in Banach spaces has been investigated in many papers, for example, in Cichon [1,2], Cramer et al. [3], Knight [4], Kubiaczyk and Szufla [5], and [6-11] and the references therein for fractionalorder differential equations in Banach spaces, and [12-14] for quadratic integral equations in reflexive Banach algebra.

The integral equations of the Volterra-Stieltjes type have been considered by many authors; for recent publications of these integral equation and the properties of the VolterraStieltjes integral equations see, for example, [15-19] and references therein.

Let $E$ be a reflexive Banach space with norm $\|\cdot\|_{E}$ and $E^{*}$ be the dual of $E$. Let $C(I, E), I=[0, T]$ be the class of strongly continuous functions $x: I \rightarrow E$ with norm $\|x\|_{C}=\sup _{t \in I}\|x(t)\|_{E}$.

Consider the delay second-order nonlinear functional integral equation of VolterraStieltjes type

$$
x(t)=f_{1}\left(t, \int_{0}^{m(t)} f_{2}(t, s, x(s)) d_{s} g(t, s)\right), t \in I
$$

and its corresponding initial value problem

$$
\begin{gathered}
\frac{d x(t)}{d t}=f_{1}\left(t, \int_{0}^{m(t)} f_{2}(t, s, x(s)) d_{s} g(t, s)\right), \quad \text { a.e }, t \in I, \\
x(0)=x_{0}
\end{gathered}
$$

where $f_{1}, f_{2}$ are weakly continuous on the bounded interval $I$ with value in the reflexive Banach space $E, g: I \times I \rightarrow R$ is nondecreasing in the second argument, $m$ is a continuous and decreasing function on $I$, and the symbol $d_{s}$ indicates integration with respect to $s$.

D. N. Sidorov [20] studied the the majorant integral equation

$$
x(t)=\int_{0}^{t} m(s) \gamma(x(s)) d s
$$


and the corresponding Cauchy problem for the differential equation

$$
\frac{d}{d t}=m(s) \gamma(x(s)), \quad x(0)=0 .
$$

He obtained sufficient conditions for the existence of a Kantorovich principal solution of the nonlinear Volterra integral equation of the second kind (4) on the half-line $[0, \infty)$ and on a finite interval $I$. He suggested a method for computing the boundary of an interval outside which the solution can blow up.

Here, we prove the existence of local solutions $x \in C(I, E)$ of the integral Equation (1) and the initial value problem (2) and (3). The sufficient conditions for the uniqueness of the solutions will be given. The continuous dependence of the solutions on the initial data $x_{o}$ and on the functions $m, f_{2}$ and $g$ will be proved. An example will be given to illustrate our results.

The following lemma and theorems will be needed in our proof (see [21,22]).

Theorem 1. Let $E$ be a normed space with $x_{0} \neq 0$. Then there exists $a \varphi \in E^{*}$ with $\|\varphi\|=1$ and $\varphi\left(x_{0}\right)=\left\|x_{0}\right\|_{E}$.

Lemma 1. A subset of a reflexive Banach space is weakly compact if and only if it is closed in the weak topology and bounded in the norm topology. Consequently, in reflexive Banach space, the subset is weakly relatively compact if and only if it is bounded in the norm topology.

Theorem 2. ( $O$ 'Regan fixed point theorem) Let $E$ be a Banach space, and let $Q$ be a nonempty, bounded, closed and convex subset of $C(I, E)$ and let $F: Q \rightarrow Q$ be weakly sequentially continuous and assume that $\{F Q(t)\}$ is relatively weakly compact in $E$ for each $t \in I$. Then, $F$ has a fixed point in the set $Q$ (see [22]).

\section{Existence of at Least One Solution}

2.1. Functional Integral Equation

Consider now the delay functional integral Equation (1) under the following assumptions:

(i) $\quad m: I \rightarrow I, m(t) \leq t$ is continuous and increasing.

(ii) $f_{1}: I \times E \rightarrow E$ is weakly continuous and weakly satisfies the Lipschitz condition

$$
\left|\varphi\left(f_{1}(t, x)-f_{1}(t, y)\right)\right| \leq k_{1}|\varphi(x-y)|, k_{1}>0, \quad \forall(t, x),(t, y) \in I \times E, \varphi \in E^{*} .
$$

(iii) $f_{2}: I \times I \times E \rightarrow E$ is weakly continuous and there exist two positive constants $k_{2}$ and $b$, such that $\left\|f_{2}(t, s, x)\right\|_{E} \leq b+k_{2}\|x\|_{E}$.

(iv) The function $g: I \times R \rightarrow R$ is continuous with

$$
\mu=\max \{\sup |g(t, m(t))|+\sup |g(t, 0)|\}, \quad \text { on } I .
$$

(v) For all $t_{1}, t_{2} \in I$ such that $t_{1}<t_{2}$, the function $s \rightarrow g\left(t_{2}, s\right)-g\left(t_{1}, s\right)$ is nondecreasing on $I$.

(vi) $g(0, s)=0$ for any $s \in I$.

(vii) $k_{1} k_{2} \mu<1$

Remark 1. From the assumption (ii) set $y=0$, we get

$$
\begin{gathered}
\left|\varphi\left(f_{1}(t, x(t))\right)\right|-\left|\varphi\left(f_{1}(t, 0)\right)\right| \leq\left|\varphi\left(f_{1}(t, x(t))-f_{1}(t, 0)\right)\right| \leq k_{1}|\varphi(x)|, \\
\left|\varphi\left(f_{1}(t, x(t))\right)\right| \leq k_{1}|\varphi(x)|+\left|\varphi\left(f_{1}(t, 0)\right)\right| .
\end{gathered}
$$

and

$$
\left|\varphi\left(f_{1}(t, x(s))\right)\right| \leq k_{1}|\varphi(x)|+f^{*}
$$

where $f^{*}=\sup _{t \in I}\left|\varphi\left(f_{1}(t, 0)\right)\right| \geq 0$. 
Definition 1. By a weak solution of (1), we mean a function $x \in C(I, E)$ that satisfies the delay composite functional integral Equation (1). This is equivalent to finding a solution $x \in C(I, E)$ of functional integral equation

$$
\varphi(x(t))=\varphi\left(f_{1}\left(t, \int_{0}^{m(t)} f_{2}(t, s, x(s)) d_{s} g(t, s)\right)\right), t \in I, \forall \varphi \in E^{*} .
$$

Now, we have the following theorem.

Theorem 3. Let the assumptions (i)-(vii) be satisfied; then, the functional Equation (1) has at least one weak solution $x \in C(I, E)$.

Proof. Define the operator $A$ by

$$
A x(t)=f_{1}\left(t, \int_{0}^{m(t)} f_{2}(t, s, x(s)) d_{s} g(t, s)\right), t \in I
$$

and the set $Q_{r}$ by

$$
Q_{r}=\left\{x \in C(I, E):\|x\|_{E} \leq r\right\}, \quad r=\frac{f^{*}+k_{1} b \mu}{1-k_{1} k_{2} \mu} .
$$

Now, let $x \in Q_{r}$, then

$$
\begin{aligned}
\|A x(t)\|_{E}=|\varphi(A x(t))| & =\left|\varphi\left(f_{1}\left(t, \int_{0}^{m(t)} f_{2}(t, s, x(s)) d_{s} g(t, s)\right)\right)\right| \\
& \leq f^{*}+k_{1}\left|\varphi\left(\int_{0}^{m(t)} f_{2}(t, s, x(s)) d_{s} g(t, s)\right)\right| \\
& \leq f^{*}+k_{1} \varphi\left(\int_{0}^{m(t)} \mid f_{2}(t, s, x(s))\right) \mid d_{s}\left(\bigvee_{z=0}^{s} g(t, z)\right) \\
& \leq f^{*}+k_{1} \int_{0}^{m(t)}\left(b+k_{2}|\varphi(x)|\right) d_{s}\left(\bigvee_{z=0}^{s} g(t, z)\right) \\
& \leq f^{*}+k_{1}\left(b+k_{2}\|x\|\right) \int_{0}^{m(t)} d_{s} g(t, s) \\
& \leq f^{*}+k_{1}\left(b+k_{2} r\right)(g(t, m(t))-g(t, 0)) \\
& \leq f^{*}+k_{1}\left(b+k_{2} r\right) \mu=r,
\end{aligned}
$$

then

$$
\|A x\|_{C}=\sup _{t \in I}|\varphi(A x(t))| \leq r .
$$

This proves that the class of functions $\{A x\}$ is uniformly bounded on $Q_{r}$.

Now we show that $A: Q_{r} \rightarrow Q_{r}$. Let $x \in Q_{r}$ and define

$$
\theta(\delta)=\sup _{x \in Q_{r}}\left\{\left|\varphi\left(f_{2}\left(t_{2}, s, x(s)\right)-f_{2}\left(t_{1}, s, x(s)\right)\right)\right|: t_{1}, t_{2} \in I, t_{1}<t_{2},\left|t_{2}-t_{1}\right|<\delta,\|x\|_{E} \leq r\right\},
$$

then, from the uniform continuity of the functions $f_{2}: I \times I \times Q_{r} \rightarrow R, f_{1}: I \times Q_{r} \rightarrow R$, and assumptions (ii) and (iii), we deduce that $\theta(\delta) \rightarrow 0$, as $\delta \rightarrow 0$ ( $\delta$ is independent of $x \in Q_{r}$ ); similarly, we have

$$
\theta_{1}(\delta)=\sup _{x \in Q_{r}}\left\{\left|\varphi\left(f_{1}\left(t_{2}, x(s)\right)-f_{1}\left(t_{1}, x(s)\right)\right)\right|: t_{1}, t_{2} \in I, t_{1}<t_{2},\left|t_{2}-t_{1}\right|<\delta,\|x\|_{E} \leq r\right\},
$$

and $\theta_{1}(\delta) \rightarrow 0$, as $\delta \rightarrow 0\left(\delta\right.$ is independent of $\left.x \in Q_{r}\right)$.

Then we have 


$$
\begin{aligned}
& \left|\varphi\left(A x\left(t_{2}\right)-A x\left(t_{1}\right)\right)\right|=\mid \varphi\left(f_{1}\left(t_{2}, \int_{0}^{m\left(t_{2}\right)} f_{2}\left(t_{2}, s, x(s)\right) d_{s} g\left(t_{2}, s\right)\right)\right. \\
& -\varphi\left(f_{1}\left(t_{2}, \int_{0}^{m\left(t_{1}\right)} f_{2}\left(t_{1}, s, x(s)\right) d_{s} g\left(t_{1}, s\right)\right)\right) \\
& +\varphi\left(f_{1}\left(t_{2}, \int_{0}^{m\left(t_{1}\right)} f_{2}\left(t_{1}, s, x(s)\right) d_{s} g\left(t_{1}, s\right)\right)\right) \\
& -\varphi\left(f_{1}\left(t_{1}, \int_{0}^{m\left(t_{1}\right)} f_{2}\left(t_{1}, s, x(s)\right) d_{s} g\left(t_{1}, s\right)\right)\right) \\
& \leq \quad k_{1}\left|\varphi\left(\int_{0}^{m\left(t_{2}\right)} f_{2}\left(t_{2}, s, x(s)\right) d_{s} g\left(t_{2}, s\right)-\int_{0}^{m\left(t_{1}\right)} f_{2}\left(t_{1}, s, x(s)\right) d_{s} g\left(t_{1}, s\right)\right)\right| \\
& +\theta_{1}(\delta) \\
& \leq \quad k_{1} \mid \varphi\left(\int_{0}^{m\left(t_{2}\right)} f_{2}\left(t_{2}, s, x(s)\right) d_{s} g\left(t_{2}, s\right)-\int_{0}^{m\left(t_{2}\right)} f_{2}\left(t_{1}, s, x(s)\right) d_{s} g\left(t_{2}, s\right)\right. \\
& \left.+\quad \int_{0}^{m\left(t_{2}\right)} f_{2}\left(t_{1}, s, x(s)\right) d_{s} g\left(t_{2}, s\right)-\int_{0}^{m\left(t_{1}\right)} f_{2}\left(t_{1}, s, x(s)\right) d_{s} g\left(t_{1}, s\right)\right) \mid+\theta_{1}(\delta) \\
& \leq \quad k_{1} \mid \varphi\left(\int_{0}^{m\left(t_{2}\right)}\left[f_{2}\left(t_{2}, s, x(s)\right)-f_{2}\left(t_{1}, s, x(s)\right)\right] d_{s} g\left(t_{2}, s\right)\right. \\
& +\quad \int_{0}^{m\left(t_{1}\right)} f_{2}\left(t_{1}, s, x(s)\right) d_{s} g\left(t_{2}, s\right)+\int_{m\left(t_{1}\right)}^{m\left(t_{2}\right)} f_{2}\left(t_{1}, s, x(s)\right) d_{s} g\left(t_{2}, s\right) \\
& \left.\left.-\quad \int_{0}^{m\left(t_{1}\right)} f_{2}\left(t_{1}, s, x(s)\right) d_{s} g\left(t_{1}, s\right)\right)\right) \mid+\theta_{1}(\delta) \\
& \leq \quad k_{1} \mid \int_{0}^{m\left(t_{2}\right)} \varphi\left(\left[f_{2}\left(t_{2}, s, x(s)\right)-f_{2}\left(t_{1}, s, x(s)\right)\right] d_{s} g\left(t_{2}, s\right)\right. \\
& +\quad \int_{0}^{m\left(t_{1}\right)} \varphi\left(f_{2}\left(t_{1}, s, x(s)\right)\right) d_{s}\left[g\left(t_{2}, s\right)-g\left(t_{1}, s\right)\right] \\
& \left.+\quad \int_{m\left(t_{1}\right)}^{m\left(t_{2}\right)} \varphi\left(f_{2}\left(t_{1}, s, x(s)\right)\right) d_{s} g\left(t_{2}, s\right)\right) \mid+\theta_{1}(\delta) \\
& \leq k_{1}\left(\int_{0}^{m\left(t_{2}\right)}\left|\varphi\left(f_{2}\left(t_{2}, s, x(s)\right)-f_{2}\left(t_{1}, s, x(s)\right)\right)\right| d_{s}\left(\bigvee_{z=0}^{s} g\left(t_{2}, z\right)\right)\right. \\
& +\quad \int_{0}^{m\left(t_{1}\right)}\left|\varphi\left(f_{2}\left(t_{1}, s, x(s)\right)\right)\right| d_{s}\left(\bigvee_{z=0}^{s}\left[g\left(t_{2}, z\right)-g\left(t_{1}, z\right)\right]\right) \\
& \left.+\quad \int_{m\left(t_{1}\right)}^{m\left(t_{2}\right)}\left|\varphi\left(f_{2}\left(t_{1}, s, x(s)\right)\right)\right| d_{s}\left(\bigvee_{z=0}^{s} g\left(t_{2}, z\right)\right)\right)+\theta_{1}(\delta) \\
& \leq k_{1} \theta(\delta) \int_{m\left(t_{1}\right)}^{m\left(t_{2}\right)} d_{s}\left(\bigvee_{z=0}^{s} g\left(t_{2}, z\right)\right) \\
& \left.+k_{1}\left(b+k_{2} r\right) \int_{0}^{m\left(t_{1}\right)} d_{s} \bigvee_{z=0}^{s}\left[g\left(t_{2}, z\right)\right)-g\left(t_{1}, z\right)\right] \\
& +k_{1}\left(b+k_{2} r\right) \int_{m\left(t_{1}\right)}^{m\left(t_{2}\right)} d_{s}\left(\bigvee_{z=0}^{s} g\left(t_{2}, z\right)\right)+\theta_{1}(\delta) \\
& \leq k_{1} \theta(\delta)\left[g\left(t_{2}, m\left(t_{2}\right)\right)-g\left(t_{2}, m\left(t_{1}\right)\right)\right] \\
& +\quad k_{1}\left(b+k_{2} r\right)\left[g\left(t_{2}, m\left(t_{1}\right)\right)-g\left(t_{1}, m\left(t_{1}\right)\right)-g\left(t_{2}, 0\right)+g\left(t_{1}, 0\right)\right] \\
& +k_{1}\left(b+k_{2} r\right)\left[g\left(t_{2}, m\left(t_{2}\right)\right)-g\left(t_{2}, m\left(t_{1}\right)\right)\right]+\theta_{1}(\delta) \text {. }
\end{aligned}
$$


Then

$$
\begin{aligned}
\left\|A x\left(t_{2}\right)-A x\left(t_{1}\right)\right\|_{E} & =\sup _{t_{1}, t_{2} \in I}\left|\varphi\left(A x\left(t_{2}\right)-A x\left(t_{1}\right)\right)\right| \\
& \leq k_{1} \theta(\delta)\left[g\left(t_{2}, m\left(t_{2}\right)\right)-g\left(t_{2}, m\left(t_{1}\right)\right)\right] \\
& +k_{1}\left(b+k_{2} r\right)\left[g\left(t_{2}, m\left(t_{1}\right)\right)-g\left(t_{1}, m\left(t_{1}\right)\right)-g\left(t_{2}, 0\right)+g\left(t_{1}, 0\right)\right] \\
& +k_{1}\left(b+k_{2} r\right)\left[g\left(t_{2}, m\left(t_{2}\right)\right)-g\left(t_{2}, m\left(t_{1}\right)\right)\right]+\theta_{1}(\delta) .
\end{aligned}
$$

Hence, we deduce that $A: Q_{r} \rightarrow Q_{r}$.

Note that $Q_{r}$ is nonempty closed, bounded, convex, and strongly equi-continuous subset of $C(I, E)$. Then according to Lemma $1,\{A Q\}$ is relatively weakly compact.

Next, to prove that the operator $A$ is weakly sequentially continuous, we take $\left\{x_{n}\right\} \subset Q_{r}$, and $\varphi\left(x_{n}\right) \rightarrow \varphi(x)$, in $Q_{r} \subseteq E$; then

$$
\varphi\left(A x_{n}(t)\right)=\varphi\left(f_{1}\left(t, \int_{0}^{m(t)} f_{2}\left(t, s, x_{n}(s)\right) d_{s} g(t, s)\right)\right)
$$

and

$$
\lim _{n \rightarrow \infty} \varphi\left(A x_{n}(t)\right)=\lim _{n \rightarrow \infty} \varphi\left(f_{1}\left(t, \int_{0}^{m(t)} f_{2}\left(t, s, x_{n}(s)\right) d_{s} g(t, s)\right)\right) .
$$

Applying Lebesgue dominated convergence Theorem (see [23]), then from assumption (iii) we have

$$
\begin{aligned}
\lim _{n \rightarrow \infty} \varphi\left(A x_{n}(t)\right) & =\varphi\left(f_{1}\left(t, \lim _{n \rightarrow \infty} \int_{0}^{m(t)} f_{2}\left(t, s, x_{n}(s)\right) d_{s} g(t, s)\right)\right) \\
& =\varphi\left(f_{1}\left(t, \int_{0}^{m(t)} f_{2}\left(t, s, \lim _{n \rightarrow \infty} x_{n}(s)\right) d_{s} g(t, s)\right)\right) \\
& =\varphi\left(f_{1}\left(t, \int_{0}^{m(t)} f_{2}(t, s, x(s)) d_{s} g(t, s)\right)\right)=\varphi(A x(t)) .
\end{aligned}
$$

Then $\varphi\left(A x_{n}(t)\right) \rightarrow \varphi(A x(t))$, which means that the operator $A$ is weakly sequentially continuous.

Since all conditions of Theorem 2 are satisfied, the operator $A$ has at least one fixed point $x \in Q_{r}$, and the integral Equation (1) has at least one weak solution $x \in C(I, E)$. This completes the proof.

\subsection{Initial Value Problem}

In order to study the existence of at least one solution of the initial value problem (2) and (3), we relax assumption (ii).

(viii) $f_{1}: I \times R \rightarrow R$ is weakly-weakly continuous and there exists $k_{1}>0$ and a weakly continuous function $a(t)$ where $a: I \rightarrow I$, such that

$$
\left|\varphi\left(f_{1}(t, y)\right)\right| \leq a(t)+k_{1}|\varphi(y)| .
$$

For the initial value problem (2) and (3), we have the following lemma.

Lemma 2. If the solution of the initial value problem (2) and (3) exists, then it can be represented by the integral equation

$$
x(t)=x_{0}+\int_{0}^{t} f_{1}\left(s, \int_{0}^{m(s)} f_{2}(s, \theta, x(\theta)) d_{\theta} g(s, \theta)\right) d s, \quad t \in I,
$$

Proof. Integrating (2), we obtain 


$$
\begin{aligned}
\int_{0}^{t} \frac{d x}{d s} & =\int_{0}^{t} f_{1}\left(s, \int_{0}^{m(s)} f_{2}(s, \theta, x(\theta)) d_{\theta} g(s, \theta)\right) d s, \\
x(t)-x(0) & =\int_{0}^{t} f_{1}\left(s, \int_{0}^{m(s)} f_{2}(s, \theta, x(\theta)) d_{\theta} g(s, \theta)\right) d s
\end{aligned}
$$

However, $x(0)=x_{0}$; then

$$
x(t)=x_{0}+\int_{0}^{t} f_{1}\left(s, \int_{0}^{m(s)} f_{2}(s, \theta, x(\theta)) d_{\theta} g(s, \theta)\right) d s .
$$

Now, differentiating (6), we get

$$
\begin{gathered}
\frac{d x}{d t}=\frac{d}{d t} x_{0}+\frac{d}{d t} \int_{0}^{t} f_{1}\left(s, \int_{0}^{m(s)} f_{2}(s, \theta, x(\theta)) d_{\theta} g(s, \theta)\right) d s, \quad t \in I \\
\frac{d x}{d t}=f_{1}\left(t, \int_{0}^{m(t)} f_{2}(t, s, x(s)) d_{s} g(t, s)\right), \quad \text { a.e. }, t \in(0, T],
\end{gathered}
$$

Now, at $t=0$ in (6) we obtain

$$
x(0)=x_{0}+\int_{0}^{0} f_{1}\left(t, \int_{0}^{m(t)} f_{2}(s, \theta, x(\theta)) d_{\theta} g(s, \theta)\right) d s .
$$

Hence

$$
x(0)=x_{0}
$$

Hence, (2), (3), and (6) are equivalent.

Now, we have the following existence theorem.

Theorem 4. Let the assumptions (i), (iii)-(vi), and (viii) be satisfied. If $k_{2} k_{1} \mu T<1$, then the initial value problem (2) and (3) has at least one solution $x \in C(I, E)$.

Proof. Define the set $Q$ by

$$
Q=\left\{x \in C(I, E):\|x\|_{E} \leq r_{1}\right\}, \quad r_{1}=\frac{\left|x_{0}\right|+a+k_{1} b \mu T}{1-k_{2} k_{1} \mu T} .
$$

where $a=\int_{0}^{t} a(s) d s$. It is clear that $Q$ is a nonempty, bounded, closed, and convex set.

Define the operator $F$ by

$$
F x(t)=x_{0}+\int_{0}^{t} f_{1}\left(s, \int_{0}^{m(s)} f_{2}(s, \theta, x(\theta)) d_{\theta} g(s, \theta)\right) d s .
$$

Let $x \in Q$, then 


$$
\begin{aligned}
\|F x(t)\|_{E}=\varphi|F x(t)| & =\left|\varphi\left(x_{0}+\int_{0}^{t} f_{1}\left(s, \int_{0}^{m(s)} f_{2}(s, \theta, x(\theta)) d_{\theta} g(s, \theta)\right) d s\right)\right| \\
& \leq\left\|x_{0}\right\|_{E}+\int_{0}^{t}\left|\varphi\left(f_{1}\left(s, \int_{0}^{m(s)} f_{2}(s, \theta, x(\theta)) d_{\theta} g(s, \theta)\right)\right)\right| d s \\
& \leq\left\|x_{0}\right\|_{E}+\int_{0}^{t}\left(\varphi(a(s))+k_{1} \int_{0}^{m(s)}\left|\varphi\left(f_{2}(s, \theta, x(\theta))\right)\right| d_{\theta} g(s, \theta)\right) d s \\
& \leq\left\|x_{0}\right\|_{E}+a+k_{1} \int_{0}^{t} \int_{0}^{m(s)}\left|\varphi\left(f_{2}(s, \theta, x(\theta))\right)\right| d_{\theta} \bigvee_{z=0}^{\theta} g(s, z) d s \\
& \leq\left\|x_{0}\right\|_{E}+a+k_{1} \int_{0}^{t} \int_{0}^{m(s)}\left(b+k_{2}|\varphi(x)|\right) d_{\theta} \bigvee_{z=0}^{\theta} g(s, z) d s \\
& \leq\left\|x_{0}\right\|_{E}+a+k_{1}\left(b+k_{2}\|x\|\right)(g(s, m(s))-g(s, 0)) T \\
& \leq\left\|x_{0}\right\|_{E}+a+k_{1}\left(b+k_{2} r_{1}\right) \mu T=r_{1} .
\end{aligned}
$$

Then

$$
\|F x\|_{C}=\sup _{t \in I}\|F x(t)\|_{E} \leq r_{1} .
$$

This proves that the class of functions $\{F x\}$ is uniformly bounded on $Q$.

Let $x \in Q, \delta>0$ be given and $t_{1}, t_{2} \in I, t_{1}<t_{2}$, such that $\left|t_{2}-t_{1}\right|<\delta$, then

$$
\begin{aligned}
&\left|\varphi\left(F x\left(t_{2}\right)-F x\left(t_{1}\right)\right)\right|=\mid \varphi\left(\int_{0}^{t_{2}} f_{1}\left(s, \int_{0}^{m(s)} f_{2}(s, \theta, x(\theta)) d_{\theta} g(s, \theta)\right) d s\right. \\
&\left.-\int_{0}^{t_{1}} f_{1}\left(s, \int_{0}^{m(s)} f_{2}(s, \theta, x(\theta)) d_{\theta} g(s, \theta)\right) d s\right) \mid \\
& \leq \mid \varphi\left(\int_{0}^{t_{1}} f_{1}\left(s, \int_{0}^{m(s)} f_{2}(s, \theta, x(\theta)) d_{\theta} g(s, \theta)\right) d s\right. \\
&+\int_{t_{1}}^{t_{2}} f_{1}\left(s, \int_{0}^{m(s)} f_{2}(s, \theta, x(\theta)) d_{\theta} g(s, \theta)\right) d s \\
&\left.-\int_{0}^{t_{1}} f_{1}\left(s, \int_{0}^{m(s)} f_{2}(s, \theta, x(\theta)) d_{\theta} g(s, \theta)\right) d s\right) \mid \\
& \leq \int_{t_{1}}^{t_{2}} \mid \varphi\left(f_{1}\left(s, \int_{0}^{m(s)} f_{2}(s, \theta, x(\theta)) \mid d_{\theta} g(s, \theta)\right) d s\right) \\
& \leq \int_{t_{1}}^{t_{2}}\left(a(s)+k_{1}\left|\int_{0}^{m(s)} \varphi\left(f_{2}(s, \theta, x(\theta))\right) d_{\theta} g(s, \theta)\right| d s\right. \\
& \leq \quad \int_{t_{1}}^{t_{2}}(a(s)\left.+k_{1} \int_{0}^{m(s)}\left|\varphi\left(f_{2}(s, \theta, x(\theta))\right)\right| d_{\theta}\left(\bigvee_{z=0}^{\theta} g(s, z)\right)\right) d s \\
& \leq \quad \int_{t_{1}}^{t_{2}}\left(a(s)+k_{1} \int_{0}^{m(s)}\left(b+k_{2}|\varphi(x)|\right) d_{\theta} g(s, \theta)\right) d s \\
& \leq \quad\left[a+k_{1}\left(b+k_{2}\|x\|\right)(g(s, m(s))-g(s, 0))\right]\left|t_{2}-t_{1}\right| \\
& \leq \quad\left[a+k_{1}\left(b+k_{2} r_{1}\right) \mu\right]\left|t_{2}-t_{1}\right| .
\end{aligned}
$$

Then

$$
\left\|F x\left(t_{2}\right)-F x\left(t_{1}\right)\right\|_{C}=\sup _{t \in I}\left|\varphi\left(F x\left(t_{2}\right)-F x\left(t_{1}\right)\right)\right| \leq\left[a+k_{1}\left(b+k_{2} r_{1}\right) \mu\right]\left|t_{2}-t_{1}\right| .
$$

The above inequality means that the operator $F$ maps $Q$ into itself.

Note that $Q$ is nonempty closed, bounded, convex, and strongly equi-continuous subset of $C(I, E)$. Then according to Lemma $1,\{F Q\}$ is relatively weakly compact. 
To prove that the operator $F$ is weakly sequentially continuous, let $\left\{x_{n}\right\} \subset Q$, and $\varphi\left(x_{n}\right) \rightarrow \varphi(x)$, in $Q \subseteq E$ then

$$
\begin{aligned}
\varphi\left(F x_{n}(t)\right) & =\varphi\left(x_{0}+\int_{0}^{t} f_{1}\left(s, \int_{0}^{m(s)} f_{2}\left(s, \theta, x_{n}(\theta)\right) d_{\theta} g(s, \theta)\right) d s\right) \\
\lim _{n \rightarrow \infty} \varphi\left(F x_{n}(t)\right) & =\lim _{n \rightarrow \infty} \varphi\left(x_{0}+\int_{0}^{t} f_{1}\left(s, \int_{0}^{m(s)} f_{2}(s, \theta, x(\theta)) d_{\theta} g(s, \theta)\right) d s\right)
\end{aligned}
$$

Applying Lebesgue dominated convergence Theorem (see[23]), then

$$
\begin{aligned}
\varphi\left(F x_{n}(t)\right) & =\varphi\left(x_{0}+\int_{0}^{t} \lim _{n \rightarrow \infty} f_{1}\left(s, \int_{0}^{m(s)} f_{2}\left(s, \theta, x_{n}(\theta)\right) d_{\theta} g(s, \theta)\right) d s\right) \\
& =\varphi\left(x_{0}+\int_{0}^{t} f_{1}\left(s, \int_{0}^{m(s)} f_{2}\left(s, \theta, \lim _{n \rightarrow \infty} x_{n}(\theta)\right) d_{\theta} g(s, \theta)\right) d s\right) \\
& =\varphi\left(x_{0}+\int_{0}^{t} f_{1}\left(s, \int_{0}^{m(s)} f_{2}\left(s, \theta, x_{0}(\theta)\right) d_{\theta} g(s, \theta)\right) d s\right)=\varphi\left(F x_{0}(t)\right) .
\end{aligned}
$$

Then $F$ is weakly sequentially continuous and by Theorem $2, F$ has a fixed point $x \in Q$ and (6) has a weak solution. Consequently, (2) and (3) have at least one weak solution $x \in C(I, E)$.

\section{Uniqueness of the Solution}

Here, we study a sufficient condition for the uniqueness of the solution $x \in C[I, E]$ of the delay functional integral Equation (1). Consider the following assumptions:

$(i i i)^{*} f_{2}: I \times I \times E \rightarrow E$ is weakly continuous and and satisfies the weakly Lipschitz condition,

$$
\left|\varphi\left(f_{2}(t, s, x)-f_{2}(t, s, y)\right)\right| \leq k_{2}|\varphi(x-y)| .
$$

From the assumption $(\text { iii })^{*}$, we have

$$
\begin{gathered}
\left|\varphi\left(f_{2}(t, s, x(s))\right)\right|-\left|\varphi\left(f_{2}(t, s, 0)\right)\right| \leq\left|\varphi\left(f_{2}(t, s, x(s))-f_{2}(t, s, 0)\right)\right| \leq k_{2}|\varphi(x)| \\
\left|\varphi\left(f_{2}(t, s, x(s))\right)\right| \leq k_{2}|\varphi(x)|+\left|\varphi\left(f_{2}(t, s, 0)\right)\right|
\end{gathered}
$$

then

$$
\left|\varphi\left(f_{2}(t, s, x(s))\right)\right| \leq k_{2}|\varphi(x)|+b,
$$

where $b=\sup _{t}\left|\varphi\left(f_{2}(t, s, 0)\right)\right|$.

Theorem 5. Let the assumptions (i) and (ii), (iii)*-(iv)-(vii) be satisfied; then the solution $x \in$ $C(I, E)$ of the integral Equation (1) is unique.

Proof. Let $x_{1}, x_{2}$ be two weakly solutions of the integral Equation (1) in the reflexive Banach space $E$ : we have 


$$
\begin{aligned}
\left|\varphi\left(x_{1}(t)-x_{2}(t)\right)\right| & =\mid \varphi\left(f_{1}\left(t, \int_{0}^{m(t)} f_{2}\left(t, s, x_{1}(s)\right) d_{s} g(t, s)\right)\right. \\
& \left.-f_{1}\left(t, \int_{0}^{m(t)} f_{2}\left(t, s, x_{2}(s)\right) d_{s} g(t, s)\right)\right) \mid \\
& \leq k_{1}\left|\varphi\left(\int_{0}^{m(t)} f_{2}\left(t, s, x_{1}(s)\right) d_{s} g(t, s)-\int_{0}^{m(t)} f_{2}\left(t, s, x_{2}(s)\right) d_{s} g(t, s)\right)\right| \\
& \leq k_{1} \int_{0}^{m(t)}\left|\varphi\left(f_{2}\left(t, s, x_{1}(s)\right)-f_{2}\left(t, s, x_{2}(s)\right)\right)\right| d_{s}\left(\bigvee_{z=0}^{s} g(t, z)\right) \\
& \leq k_{1} k_{2} \int_{0}^{m(t)}\left|\varphi\left(x_{1}(s)-x_{2}(s)\right)\right| d_{s}\left(\bigvee_{z=0}^{s} g(t, z)\right) \\
& \leq k_{1} k_{2}\left\|x_{1}-x_{2}\right\|_{C} \int_{0}^{m(t)} d_{s} g(t, s),
\end{aligned}
$$

therefore

$$
\begin{aligned}
\left\|x_{1}-x_{2}\right\|_{C} & \leq k_{1} k_{2}\left\|x_{1}-x_{2}\right\|_{C}[g(t, m(t))-g(t, 0)] \\
& \leq k_{1} k_{2} \mu\left\|x_{1}-x_{2}\right\|_{C}
\end{aligned}
$$

and

$$
\left(1-k_{1} k_{2} \mu\right)\left\|x_{1}-x_{2}\right\|_{C} \leq 0 .
$$

However, $k_{1} k_{2} \mu<1$; then

$$
\left\|x_{1}-x_{2}\right\|_{C}=0 \text {. }
$$

Thus, $x_{1}=x_{2}$ and the solution of the functional integral Equation (1) is unique.

To study the uniqueness of the solution of the initial value problem (2) and (3), we replace the assumption (viii) by

$\left(\right.$ viii) ${ }^{*} f_{1}: I \times R \rightarrow R$ is weakly continuous and satisfying weakly Lipschitz condition

$$
\left|\varphi\left(f_{1}(t, x)-f_{1}(t, y)\right)\right| \leq k_{1}|\varphi(x-y)|, k_{1}>0, \quad \forall(t, x),(t, y) \in I \times E
$$

Theorem 6. Let the assumptions (i), (iii)-(vi), (viii)* be satisfied. If $k_{1} k_{2} \mu T<1$, then the weak solution $x \in C(I, E)$ of the initial value problem (2) and (3) is unique.

Proof. Let $x_{1}=x_{2}$ be two weak solutions of the functional integral Equation (6), we get

$$
\begin{aligned}
\left\|x_{1}(t)-x_{2}(t)\right\|_{E}=\left|\varphi\left(x_{1}(t)-x_{2}(t)\right)\right| & =\mid \varphi\left(x_{0}+\int_{0}^{t} f_{1}\left(s, \int_{0}^{m(s)} f_{2}\left(s, \theta, x_{1} \theta(s)\right) d_{\theta} g(s, \theta)\right) d s\right. \\
& \left.-\left(x_{0}+\int_{0}^{t} f_{1}\left(s, \int_{0}^{m(s)} f_{2}\left(s, \theta, x_{2} \theta(s)\right) d_{\theta} g(s, \theta)\right) d s\right)\right) \mid \\
& \leq k_{1} \int_{0}^{t} \mid \varphi\left(\int_{0}^{m(s)} f_{2}\left(s, \theta, x_{1}(\theta)\right) d_{\theta} g(s, \theta)\right. \\
& \left.-\int_{0}^{m(s)} f_{2}\left(s, \theta, x_{2}(\theta)\right) d_{\theta} g(s, \theta)\right) \mid d s \\
& \leq k_{1} k_{2} \int_{0}^{t} \int_{0}^{m(s)}\left|\varphi\left(x_{1}(\theta)-x_{2}(\theta)\right)\right| d_{\theta}\left(\bigvee_{z=0}^{\theta} g(s, z) d s\right. \\
& \leq k_{1} k_{2}\left\|x_{1}-x_{2}\right\|_{C} \int_{0}^{t} \int_{0}^{m(s)} d_{\theta} g(s, \theta) d s \\
& \leq k_{1} k_{2}\left\|x_{1}-x_{2}\right\|_{C} \int_{0}^{t}[g(s, m(s))-g(s, 0)] d s,
\end{aligned}
$$


thus

$$
\begin{aligned}
\left\|x_{1}-x_{2}\right\|_{C} & \leq k_{1} k_{2} \mu\left\|x_{1}-x_{2}\right\|_{C} \int_{0}^{t} d s \\
& \leq k_{1} k_{2} \mu T\left\|x_{1}-x_{2}\right\|_{C} \\
\left\|x_{1}-x_{2}\right\|_{C}\left[1-k_{1} k_{2} \mu T\right] & \leq 0 .
\end{aligned}
$$

However, $k_{1} k_{2} \mu T<1$, then

$$
\left\|x_{1}-x_{2}\right\|_{C}=0
$$

This means that

$$
x_{1}-x_{2}=0 \Rightarrow x_{1}=x_{2} .
$$

Thus, the solution of the integral Equation (6) is unique; consequently, the solution of the initial value problem (2) and (3) is unique.

\section{Continuous Dependence}

Definition 2. The solutions of the functional integral Equation (1) and the initial value problem (2) and (3) depend continuously on the delay function $m$ if $\forall \epsilon>0, \exists \delta>0$, such that

$$
\left|m(t)-m^{*}(t)\right| \leq \delta \Rightarrow\left\|x-x^{*}\right\|_{C} \leq \epsilon .
$$

Theorem 7. Let the assumptions of Theorem 5 be satisfied; then the solution of the functional integral Equation (1) depends continuously on the delay function $\mathrm{m}$.

Proof. Let $\delta>0$ be given such that $\left|m(t)-m^{*}(t)\right| \leq \delta, \forall t \in I$; we obtain

$$
\begin{aligned}
\left|\varphi\left(x(t)-x^{*}(t)\right)\right| \leq & \varphi\left(f_{1}\left(t, \int_{0}^{m(t)} f_{2}(t, s, x(s)) d_{s} g(t, s)\right)-f_{1}\left(t, \int_{0}^{m^{*}(t)} f_{2}\left(t, s, x^{*}(s)\right) d_{s} g(t, s)\right)\right) \\
& \leq k_{1}\left|\int_{0}^{m(t)} \varphi\left(f_{2}(t, s, x(s)) d_{s} g(t, s)\right)-\int_{0}^{m^{*}(t)} \varphi\left(f_{2}\left(t, s, x^{*}(s)\right) d_{s} g(t, s)\right)\right| \\
& \leq k_{1} \mid \int_{0}^{m(t)} \varphi\left(f_{2}(t, s, x(s)) d_{s} g(t, s)\right)-\int_{0}^{m(t)} \varphi\left(f_{2}\left(t, s, x^{*}(s)\right) d_{s} g(t, s)\right) \\
& +\int_{0}^{m(t)} \varphi\left(f_{2}\left(t, s, x^{*}(s)\right) d_{s} g(t, s)\right)-\int_{0}^{m^{*}(t)} \varphi\left(f_{2}\left(t, s, x^{*}(s)\right) d_{s} g(t, s)\right) \mid \\
& \leq k_{1} \int_{0}^{m(t)}\left|\varphi\left(f_{2}(t, s, x(s))-f_{2}\left(t, s, x^{*}(s)\right)\right)\right| d_{s}\left(\bigvee_{z=0}^{s} g(t, z)\right) \\
& +\int_{m^{*}(t)}^{m(t)}\left|\varphi\left(f_{2}\left(t, s, x^{*}(s)\right)\right)\right| d_{s}\left(\bigvee_{z=0}^{s} g(t, z)\right. \\
& \leq k_{1}\left[k_{2} \int_{0}^{m(t)}\left|\varphi\left(x(s)-x^{*}(s)\right)\right| d_{s} g(t, s)+\int_{m^{*}(t)}^{m(t)}\left(b+k_{2}|\varphi(x)|\right) d_{s} g(t, s)\right] \\
\leq & k_{1}\left[k_{2}\left\|x-x^{*}\right\|_{C}\left[g\left(t, m^{*}(t)\right)-g(t, 0)\right]+\left(b+k_{2} r\right) \int_{m^{*}(t)}^{m(t)} d_{s} g(t, s)\right],
\end{aligned}
$$

and hence

$$
\left\|x-x^{*}\right\|_{C} \leq k_{1} k_{2} \mu\left\|x-x^{*}\right\|_{C}+k_{1}\left(b+k_{2} r\right)\left|g(t, m(t))-g\left(t, m^{*}(t)\right)\right| .
$$

However, from the continuity of $g$, we have

$$
\left|m(t)-m^{*}(t)\right| \leq \delta \Rightarrow\left|g(t, m(t))-g\left(t, m^{*}(t)\right)\right|<\epsilon_{1},
$$


then

$$
\begin{aligned}
\left\|x-x^{*}\right\|_{C} & \leq k_{1} k_{2}\left\|x-x^{*}\right\|_{C} \mu+k_{1}\left(b+k_{2} r\right) \epsilon_{1} \\
\left\|x-x^{*}\right\|_{C}\left(1-k_{1} k_{2} \mu\right) & \leq k_{1}\left(b+k_{2} r\right) \epsilon_{1} \\
\left\|x-x^{*}\right\|_{C} & \leq \frac{k_{1}\left(b+k_{2} r\right) \epsilon_{1}}{1-k_{1} k_{2} \mu}=\epsilon .
\end{aligned}
$$

This completes the proof.

Theorem 8. Let the assumptions of Theorem 6 be satisfied; then the solution of the initial value problem (2) and (3) depends continuously on the delay function $m$.

Proof. Let $\delta>0$ be given such that $\left|m(t)-m^{*}(t)\right| \leq \delta, \forall t \geq 0$; then

$$
\begin{aligned}
\left\|x(t)-x^{*}(t)\right\|_{E} & \leq \varphi\left(\int_{0}^{t} f_{1}\left(s, \int_{0}^{m(s)} f_{2}(s, \theta, x(\theta)) d_{\theta} g(s, \theta)\right) d s\right) \\
& \left.-\quad \int_{0}^{t} f_{1}\left(s, \int_{0}^{m^{*}(s)} f_{2}\left(s, \theta, x^{*}(\theta)\right) d_{\theta} g(s, \theta)\right) d s\right) \\
\leq & k_{1}\left|\int_{0}^{t} \int_{0}^{m(s)} \varphi\left(f_{2}(s, \theta, x(\theta)) d_{\theta} g(s, \theta)\right)-\int_{0}^{m^{*}(t)} \varphi\left(f_{2}\left(s, \theta, x^{*}(\theta)\right) d_{\theta} g(s, \theta)\right)\right| d s \\
\leq & k_{1} \int_{0}^{t} \mid \int_{0}^{m(s)} \varphi\left(f_{2}(s, \theta, x(\theta)) d_{\theta} g(s, \theta)-\int_{0}^{m(s)} \varphi\left(f_{2}\left(\left(s, \theta, x^{*}(\theta)\right) d_{\theta} g(s, \theta)\right) d s\right.\right. \\
& +\quad \int_{0}^{m(s)} \varphi\left(f_{2}\left(s, \theta, x^{*}(\theta)\right) d_{\theta} g(s, \theta)\right)-\int_{0}^{m^{*}(s)} \varphi\left(f_{2}\left(s, \theta, x^{*}(\theta)\right) d_{\theta} g(s, \theta)\right) \mid d s \\
\leq & k_{1} \int_{0}^{t}\left(\int_{0}^{m(s)}\left|\varphi\left(f_{2}(s, \theta, x(\theta))-f_{2}\left(s, \theta, x^{*}(\theta)\right)\right)\right|\left(\bigvee_{z=0}^{\theta} g(s, z)\right) d s\right. \\
& +\int_{m^{*}(s)}^{m(s)}\left|\varphi\left(f_{2}\left(s, \theta, x^{*}(\theta)\right)\right)\right|\left(\bigvee_{z=0}^{\theta} g(s, z)\right) d s \\
\leq \quad & k_{1}\left(k_{2} \int_{0}^{t} \int_{0}^{m(s)}\left|\varphi\left(x(\theta)-x^{*}(\theta)\right)\right|\left(\bigvee_{z=0}^{\theta} g(s, z)\right) d s+\int_{m^{*}(s)}^{m(s)}\left(b+k_{2}|\varphi(x)|\right)\left(\bigvee_{z=0} g(s, z)\right) d s\right) \\
\leq \quad & k_{1}\left(k_{2}\left\|x-x^{*}\right\|_{C} \int_{0}^{t}\left[\int_{0}^{m^{*}(s)} d_{\theta} g(s, \theta)\right)+\int_{m^{*}(t)}^{m(s)}\left(b+k_{2}|\varphi(x)|\right) d_{\theta} g(s, \theta)\right) d s \\
\leq \quad & k_{1} k_{2}\left\|x-x^{*}\right\|_{C} \int_{0}^{t}\left([g(s, m(\theta))-g(s, 0)]+k_{1}\left(b+k_{2} r_{1}\right) \int_{m^{*}(s)}^{m(s)} d_{\theta} g(s, \theta)\right) d s . \\
\leq \quad & k_{3} k_{2} \mu T\left\|x-x^{*}\right\|_{C}+k_{1} T\left(b+k_{2} r_{1}\right)\left[g(s, m(\theta))-g\left(s, m^{*}(\theta)\right)\right] .
\end{aligned}
$$

However, from the continuity of $g$, we have

$$
\left|m(s)-m^{*}(s)\right| \leq \delta \Rightarrow\left|g(s, m(\theta))-g\left(s, m^{*}(\theta)\right)\right|<\epsilon_{1}
$$

then

$$
\begin{aligned}
\left\|x-x^{*}\right\|_{C} & \leq k_{1} k_{2} \mu T\left\|x-x^{*}\right\|_{C}+k_{1} T\left(b+k_{1} r_{2}\right) \epsilon_{1} \\
\left\|x-x^{*}\right\|_{C}\left(1-k_{1} k_{2} \mu T\right) & \leq k_{1} T\left(b+k_{2} r_{1}\right) \epsilon_{1} \\
\left\|x-x^{*}\right\|_{C} & \leq \frac{k_{1} T\left(b+k_{2} r_{1}\right) \epsilon_{1}}{1-k_{1} k_{2} \mu T}=\epsilon .
\end{aligned}
$$

This completes the proof.

Definition 3. The solutions of the functional integral Equation (1) and the initial value problem (2) and (3) depend continuously on the function $f_{2}(t, s, x(s))$ if $\forall \epsilon>0, \exists \delta>0$, such that

$$
\left|\varphi\left(f_{2}(t, s, x(s))-f_{2}^{*}(t, s, x(s))\right)\right| \leq \delta \Rightarrow\left\|x-x^{*}\right\| \leq \epsilon .
$$


Now we will study the continuous dependence on the function $f_{2}(t, s, x(s))$.

Theorem 9. Let the assumptions of Theorem 5 be satisfied; then the solution of the functional integral Equation (1) depends continuously on the function $f_{2}$.

Proof. Let

$$
\left|\varphi\left(f_{2}(t, s, x(s))-f_{2}^{*}(t, s, x(s))\right)\right| \leq \delta,
$$

then we get

$$
\begin{aligned}
\left\|x(t)-x^{*}(t)\right\|_{E} & =\varphi\left(f_{1}\left(t, \int_{0}^{m(t)} f_{2}(t, s, x(s)) d_{s} g(t, s)\right)-f_{1}\left(t, \int_{0}^{m(t)} f_{2}^{*}\left(t, s, x^{*}(s)\right) d_{s} g(t, s)\right)\right) \\
& \leq k_{1}\left|\int_{0}^{m(t)} \varphi\left(f_{2}(t, s, x(s))\right) d_{s} g(t, s)-\int_{0}^{m(t)} \varphi\left(f_{2}^{*}\left(t, s, x^{*}(s)\right)\right) d_{s} g(t, s)\right| \\
& \leq k_{1} \mid \int_{0}^{m(t)} \varphi\left(f_{2}(t, s, x(s))\right) d_{s} g(t, s)-\int_{0}^{m(t)} \varphi\left(f_{2}^{*}(t, s, x(s))\right) d_{s} g(t, s) \\
& +\int_{0}^{m(t)} \varphi\left(f_{2}^{*}(t, s, x(s))\right) d_{s} g(t, s)-\int_{0}^{m(t)} \varphi\left(f_{2}^{*}\left(t, s, x^{*}(s)\right)\right) d_{s} g(t, s) \mid \\
& \leq k_{1}\left(\int_{0}^{m(t)}\left|\varphi\left(f_{2}(t, s, x(s))-f_{2}^{*}(t, s, x(s))\right)\right| d_{s}\left(\bigvee_{z=0}^{s} g(t, z)\right)\right. \\
& \left.+\int_{0}^{m(t)}\left|\varphi\left(f_{2}^{*}(t, s, x(s))-f_{2}^{*}\left(t, s, x^{*}(s)\right)\right)\right|\left(\bigvee_{z=0}^{s} g(t, z)\right)\right) \\
\leq & k_{1}\left[\delta \int_{0}^{m(t)} d_{s} g(t, s)+k_{2} \int_{0}^{m(t)}\left|\varphi\left(x(s)-x^{*}(s)\right)\right| d_{s} g(t, s)\right] \\
\leq & k_{1} \delta[g(t, m(t))-g(t, 0)]+k_{1} k_{2}\left\|x-x^{*}\right\|_{C}[g(t, m(t))-g(t, 0)] \\
\leq & k_{1} \delta \mu+k_{1} k_{2} \mu\left\|x-x^{*}\right\|_{C}
\end{aligned}
$$

then

$$
\begin{aligned}
\left\|x-x^{*}\right\|_{C}\left[1-k_{1} k_{2} \mu\right] & \leq k_{1} \delta \mu \\
\left\|x-x^{*}\right\|_{C} & \leq \frac{k_{1} \delta \mu}{1-k_{1} k_{2} \mu}=\epsilon .
\end{aligned}
$$

This completes the proof.

Theorem 10. Let the assumptions of Theorem 6 be satisfied; then the solution of initial value problem (2) and (3) depends continuously on the function $f_{2}$.

Proof. Let

$$
\left|\varphi\left(f_{2}(t, s, x(s))-f_{2}^{*}(t, s, x(s))\right)\right| \leq \delta,
$$




\section{then we get}

$$
\begin{aligned}
\left\|x(t)-x^{*}(t)\right\|_{E} & =\varphi\left(\int_{0}^{t} f_{1}\left(s, \int_{0}^{m(s)} f_{2}(s, \theta, x(\theta)) d_{s} g(t, s)\right) d s\right. \\
& \left.-\int_{0}^{t} f_{1}\left(s, \int_{0}^{m(s)} f_{2}^{*}\left(s, \theta, x^{*}(\theta)\right) d_{\theta} g(s, \theta) d s\right)\right) \\
& \leq k_{1}\left|\int_{0}^{t}\left[\int_{0}^{m(s)} \varphi\left(f_{2}(s, \theta, x(\theta))\right) d_{\theta} g(s, \theta)-\int_{0}^{m(s)} \varphi\left(f_{2}^{*}\left(s, \theta, x^{*}(\theta)\right)\right) d_{\theta} g(s, \theta)\right] d s\right| \\
& \leq k_{1} \mid \int_{0}^{t}\left[\int_{0}^{m(s)} \varphi\left(f_{2}(s, \theta, x(\theta))\right) d_{\theta} g(s, \theta)-\int_{0}^{m(s)} \varphi\left(f_{2}^{*}(s, \theta, x(\theta))\right) d_{\theta} g(s, \theta)\right] \\
& \left.+\int_{0}^{m(s)} \varphi\left(f_{2}^{*}(s, \theta, x(\theta))\right) d_{\theta} g(s, \theta)-\int_{0}^{m(s)} \varphi\left(f_{2}^{*}\left(s, \theta, x^{*}(\theta)\right)\right) d_{\theta} g(s, \theta)\right] d s \mid \\
& \leq k_{1} \int_{0}^{t}\left[\int_{0}^{m(s)}\left|\varphi\left(f_{2}(s, \theta, x(\theta))-f_{2}^{*}(s, \theta, x(\theta))\right)\right| d_{\theta}\left(\bigvee_{z=0}^{\theta} g(s, z)\right)\right. \\
& \left.+\int_{0}^{m(s)}\left|\varphi\left(f_{2}^{*}(s, \theta, x(\theta))-f_{2}^{*}\left(s, \theta, x^{*}(\theta)\right)\right)\right| d_{\theta}\left(\bigvee_{z=0}^{\theta} g(s, z)\right)\right] d s \\
\leq & \left.k_{1} \int_{0}^{t}\left[\delta \int_{0}^{m(s)} d_{\theta} g(s, \theta)+k_{2} \int_{0}^{m(s)} \mid \varphi\left(x(\theta)-x^{*}(\theta)\right)\right) \mid d_{\theta} g(s, \theta)\right] d s \\
\leq & k_{1} \delta T[g(s, m(s))-g(s, 0)]+k_{1} k_{2} T\left\|x-x^{*}\right\|[g(t, m(s))-g(s, 0)] \\
\leq & k_{1} \delta \mu T+k_{1} k_{2} \mu T\left\|x-x^{*}\right\|_{C},
\end{aligned}
$$

then

$$
\begin{aligned}
\left\|x-x^{*}\right\|_{C}\left[1-k_{1} k_{2} \mu T\right] & \leq k_{1} \delta \mu T \\
\left\|x-x^{*}\right\|_{C} & \leq \frac{k_{1} \delta \mu T}{1-k_{1} k_{2} \mu T}=\epsilon .
\end{aligned}
$$

This completes the proof.

Definition 4. The solution of the initial value problem (2) and (3) depends continuously on the initial data $x_{0}$ if $\forall \epsilon>0, \exists \delta>0$, such that

$$
\left|x_{0}-x_{0}^{*}\right| \leq \delta \Rightarrow\left\|x-x^{*}\right\|_{C} \leq \epsilon .
$$

Theorem 11. Let the assumptions of Theorem 6 be satisfied; then, the solution of the initial value problem (2) and (3) depends continuously on the initial data $x_{0}$.

Proof. Let

$$
\begin{aligned}
& \left|x_{0}-x_{0}^{*}\right| \leq \delta . \\
\left\|x(t)-x^{*}(t)\right\|_{E} & =\varphi\left(x_{0}+\int_{0}^{t} f_{1}\left(s, \int_{0}^{m(s)} f_{2}(s, \theta, x(\theta)) d_{\theta} g(s, \theta)\right) d s\right. \\
& \left.-\quad x_{0}^{*}+\int_{0}^{t} f_{1}\left(s, \int_{0}^{m(t)} f_{2}(s, \theta, x(\theta)) d_{\theta} g(s, \theta)\right) d s\right) \\
\leq & \left|x_{0}-x_{0}^{*}\right|+k_{1} \mid \int_{0}^{t}\left[\int_{0}^{m(s)} \varphi\left(f_{2}(s, \theta, x(\theta))\right) d_{\theta} g(s, \theta)\right. \\
& \left.-\int_{0}^{m(t)} \varphi\left(f_{2}\left(s, \theta, x^{*}(\theta)\right) d_{\theta} g(s, \theta)\right)\right] d s \mid \\
\leq & \delta+k_{1} k_{2} \int_{0}^{t}\left[\int_{0}^{m(s)}\left|\varphi\left(x(\theta)-x^{*}(\theta)\right)\right| d_{\theta} g(s, \theta)\right] d s \\
\leq & \delta+k_{1} k_{2}\left\|x-x^{*}\right\| \int_{0}^{t}\left[\int_{0}^{m(s)} d_{\theta} g(s, \theta)\right] d s \\
\leq & \delta+k_{1} k_{2}\left\|x-x^{*}\right\|_{C} \int_{0}^{t}[g(s, m(s))-g(s, 0)] d s \\
\leq & \delta+k_{1} k_{2} T \mu\left\|x-x^{*}\right\|_{C}
\end{aligned}
$$


then

$$
\begin{aligned}
\left\|x-x^{*}\right\|_{C}\left(1-k_{1} k_{2} T \mu\right) & \leq \delta \\
\left\|x-x^{*}\right\|_{C} & \leq \frac{\delta}{1-k_{1} k_{2} T \mu}=\epsilon .
\end{aligned}
$$

This completes the proof.

Definition 5. The solutions of the functional integral Equation (1) and the initial value problem (2) and (3) depend continuously on the function $g(t, s)$ if $\forall \epsilon>0, \exists \delta>0$, such that

$$
\left|g(t, s)-g^{*}(t, s)\right| \leq \delta \Rightarrow\left\|x-x^{*}\right\| \leq \epsilon .
$$

Theorem 12. Let the assumptions of Theorem 5 be satisfied; then the solution of the functional integral Equation (1) depends continuously on the function $g(t, s)$.

Proof. Let $\delta>0$ be given such that $\left|g(t, s)-g^{*}(t, s)\right| \leq \delta, \forall t \geq 0$, then

$$
\begin{aligned}
\left\|x(t)-x^{*}(t)\right\|_{E} & \leq \varphi\left(f_{1}\left(t, \int_{0}^{m(t)} f_{2}(t, s, x(s)) d_{s} g(t, s)\right)\right)-\varphi\left(f_{1}\left(t, \int_{0}^{m(t)} f_{2}\left(t, s, x^{*}(s)\right) d_{s} g^{*}(t, s)\right)\right) \\
& \leq k_{1}\left|\int_{0}^{m(t)} \varphi\left(f_{2}(t, s, x(s)) d_{s} g(t, s)\right)-\int_{0}^{m(t)} \varphi\left(f_{2}\left(t, s, x^{*}(s)\right) d_{s} g^{*}(t, s)\right)\right| \\
& \leq k_{1} \mid \int_{0}^{m(t)} \varphi\left(f_{2}(t, s, x(s)) d_{s} g(t, s)\right)-\int_{0}^{m(t)} \varphi\left(f_{2}\left(t, s, x^{*}(s)\right) d_{s} g(t, s)\right) \\
& +\int_{0}^{m(t)} \varphi\left(f_{2}\left(t, s, x^{*}(s)\right) d_{s} g(t, s)\right)-\int_{0}^{m(t)} \varphi\left(f_{2}\left(t, s, x^{*}(s)\right) d_{s} g^{*}(t, s)\right) \mid \\
& \leq k_{1} \mid \int_{0}^{m(t)} \varphi\left(f_{2}(t, s, x(s))-\left(f_{2}\left(t, s, x^{*}(s)\right)\right) d_{s} g(t, s)\right) \\
& +\int_{0}^{m(t)} \varphi\left(f_{2}\left(t, s, x^{*}(s)\right) d_{s}(g(t, s))-g^{*}(t, s)\right) \mid \\
& \left.\leq k_{1} k_{2}\left|\int_{0}^{m(t)}\right| \varphi\left(x(t)-x^{*}(t)\right) \mid d_{s} g(t, s)\right)+\int_{0}^{m(t)}\left(b+k_{2}|\varphi(x)|\right) d_{s}\left(g(t, s)-g^{*}(t, s)\right) \\
& \left.\left.\leq k_{1} k_{2} \mu\left\|x-x^{*}\right\|_{C}+\left(b+k_{2}\|x\|\right)\left((g(t, m(t)))-g^{*}(t, m(t))\right)-(g(t, 0))-g^{*}(t, 0)\right)\right) \\
& \leq k_{1} k_{2} \mu\left\|x-x^{*}\right\|_{C}+\left(b+k_{2} r\right) 2 \delta,
\end{aligned}
$$

then

$$
\begin{aligned}
\left\|x-x^{*}\right\|_{C}\left[1-k_{1} k_{2} \mu\right] & \leq\left(b+k_{2} r\right) 2 \delta \\
\left\|x-x^{*}\right\|_{C} & \leq \frac{\left(b+k_{2} r\right) 2 \delta}{1-k_{1} k_{2} \mu}=\epsilon .
\end{aligned}
$$

This completes the proof.

Theorem 13. Let the assumptions of Theorem 6 be satisfied then the solution of initial value problem (2) and (3) depends continuously on the function $g(t, s)$.

Proof. Let

$$
\left|g(t, s)-g^{*}(t, s)\right| \leq \delta,
$$

then we get 


$$
\begin{aligned}
\left\|x(t)-x^{*}(t)\right\|_{E} & =\varphi\left(\int_{0}^{t} f_{1}\left(s, \int_{0}^{m(s)} f_{2}(s, \theta, x(\theta)) d_{s} g(t, s)\right) d s\right. \\
& \left.-\int_{0}^{t} f_{1}\left(s, \int_{0}^{m(s)} f_{2}\left(s, \theta, x^{*}(\theta)\right) d_{\theta} g^{*}(s, \theta) d s\right)\right) \\
\leq & k_{1}\left|\int_{0}^{t}\left[\int_{0}^{m(s)} \varphi\left(f_{2}(s, \theta, x(\theta))\right) d_{\theta} g(s, \theta)-\int_{0}^{m(s)} \varphi\left(f_{2}\left(s, \theta, x^{*}(\theta)\right)\right) d_{\theta} g^{*}(s, \theta)\right] d s\right| \\
& \leq k_{1} \mid \int_{0}^{t}\left[\int_{0}^{m(s)} \varphi\left(f_{2}(s, \theta, x(\theta))\right) d_{\theta} g(s, \theta)-\int_{0}^{m(s)} \varphi\left(f_{2}\left(s, \theta, x^{*}(\theta)\right)\right) d_{\theta} g(s, \theta)\right] \\
& \left.+\int_{0}^{m(s)} \varphi\left(f_{2}\left(s, \theta, x^{*}(\theta)\right)\right) d_{\theta} g(s, \theta)-\int_{0}^{m(s)} \varphi\left(f_{2}\left(s, \theta, x^{*}(\theta)\right)\right) d_{\theta} g^{*}(s, \theta)\right] d s \mid \\
& \leq k_{1} \mid \int_{0}^{t}\left[\int_{0}^{m(s)} \varphi\left(f_{2}(s, \theta, x(\theta))-f_{2}\left(s, \theta, x^{*}(\theta)\right)\right) d_{\theta} g(s, \theta)\right. \\
& \left.+\int_{0}^{m(s)} \varphi\left(f_{2}\left(s, \theta, x^{*}(\theta)\right)\right) d_{\theta}\left(g(s, \theta)-g^{*}(s, \theta)\right)\right] d s \mid \\
& \leq k_{1} \mid \int_{0}^{t}\left[k_{2} \int_{0}^{m(s)}\left|\varphi\left(x(\theta)-x^{*}(\theta)\right)\right| d_{\theta} g(s, \theta)\right. \\
& +\int_{0}^{m(s)}\left(b+k_{2}\left|\varphi\left(x^{*}(\theta)\right)\right| d_{\theta}\left(g(s, \theta)-g^{*}(s, \theta)\right)\right] d s \mid \\
& \leq k_{1} \int_{0}^{t}\left[k_{2} \mu\left\|x-x^{*}\right\|_{C} \quad\|\quad\| x-x^{*} \|_{C} \leq \frac{k_{1}\left(b+k_{2} r\right) 2 \delta T}{1-k_{1} k_{2} \mu T}=\epsilon .\right. \\
& \left.\left(b+k_{2} r\right)\left[\left(g(s, m(s))-g^{*}(s, m(s))\right)-\left(g(s, 0)-g^{*}(s, 0)\right)\right]\right] d s \\
& \leq k_{1} k_{2} \mu T\left\|x-x^{*}\right\|_{C}+k_{1}\left(b+k_{2} r\right) 2 \delta, \\
&
\end{aligned}
$$

This completes the proof.

\section{Examples}

Example 1. Consider the delay functional integral equation

$$
x(t)=t+\frac{1}{3} \int_{0}^{t}\left(t^{2}+t s+t x^{2}(s)\right) d s, t \in I
$$

Obviously, this equation is a special case of (1). Indeed, if we put

$$
\begin{gathered}
f_{1}(t, x)=t+\frac{1}{3} x \\
f_{2}(t, s, x)=t+s+x^{2}(s)
\end{gathered}
$$

and $g(t, s)=t(t+s-1) \Rightarrow d_{s} g(t, s)=t d s, m(t)=t$.

It is clear that the functions $f_{1}, f_{2}, g$ satisfy the assumptions of Theorem 5 ; then, the functional integral Equation (7) has one weak solution $x \in C(I, E)$.

Example 2. Consider the initial value problem

$$
\begin{gathered}
\frac{d x(t)}{d t}=t+\frac{t^{2}}{2}+\int_{0}^{m(t)} \frac{t}{t+s}\left(t+s+x^{2}(s)\right) d s, \quad \text { a.e }, t \in I, \\
x(0)=x_{0}
\end{gathered}
$$




$$
\begin{aligned}
& \text { where } f_{1}(t, x)=t+\frac{t^{2}}{2}+x, f_{2}(t, s, x)=t+s+x^{2}(s) \text { and } \\
& \qquad g(t, s)= \begin{cases}t \ln \frac{t+s}{t}, & \text { for } t \in(0, T], s \in I, \\
0, & \text { for } t=0, s \in I,\end{cases}
\end{aligned}
$$

It is clear that this problem is a special case of the initial value problems (2) and (3), Where $d_{s} g(t, s)=\frac{t}{t+s} d s, f_{1}, f_{2}, g$ satisfy the assumptions (iii)-(vi) and (viii) in Theorem 6; then the initial value problem (8) and (9) has a one weak solution $x \in C(I, E)$.

\section{Conclusions}

The theory of differential equations in abstract Banach spaces has been established by some authors from different viewpoints, for example, [24-27]. Here we have proved the existence of solutions $x \in C(I, E)$ for the delay functional integral Equation (1) and its corresponding initial value problem (2) and (3) which have been studied in a reflexive Banach space $E$. The continuous solutions of (1)-(3) on the delay function $m$ and the function $f_{2}$ and the continuous solution of (2) and (3) on the initial data $x_{0}$ have been proved. As an application, two examples are given.

Author Contributions: Conceptualization, A.M.A.E.-S. and Y.M.Y.O. All authors have read and agreed to the published version of the manuscript.

Funding: This research received no external funding.

Institutional Review Board Statement: Not applicable.

Informed Consent Statement: Not applicable.

Data Availability Statement: Not applicable.

Acknowledgments: Authors are grateful to referees for their useful comments and remarks that helped to improve this work.

Conflicts of Interest: The authors declare no conflict of interest.

\section{References}

1. Cichoń, M. Weak solutions of ordinary differential equations in Banach spaces. Discuss. Differ. Inc. Control Optimal. 1995, 15, 5-14.

2. Cichoń, M.; Kubiaczyk, I.; Sikorska-Nowak, A.; Yantir, A.,Weak solutions for dynamic Cauchy problem in Banach spaces. Nonlinear Anal. 2009, 71, 2936-2943. [CrossRef]

3. Cramer, E.; Lakshmiksntham, V.; Mitchell, A.R. On the existence of weak solutions of differential equations in nonreflexive Banach spaces. Nonlinear Anal. 1978, 2, 259-262 [CrossRef]

4. Knight, W.J. Solutions of differential equations in Banach spaces. Duke Math. J. 1974, 41, 437-442. [CrossRef]

5. Kubiaczyk, I.; Szufla, S. Kneser's theorem for weak solutions of ordinary differential equations in Banach spaces. Publ. Inst. Math. 1982, 32, 99-103.

6. Agarwal, R.P.; Lupulescu, V.; O’Regan, D.; Rahman, G.U. Nonlinear fractional differential equations in nonreflexive Banach spaces and fractional calculus. Adv. Differ. Equ. 2015, 2015, 112. [CrossRef]

7. Agarwal, R.P.; Lupulescu, V.; O’Regan, D.; Rahman, G.U. Weak solutions for fractional differential equations in nonreflexive Banach spaces via Riemann-Pettis integrals. Math. Nachr. 2016, 289, 395-409. [CrossRef]

8. Hashem, H.H.G.; El-Sayed, A.M.A.; Aleniz, M.A. Weak and pseudo-solutions of an arbitrary (fractional) orders differential equation in nonreflexive Banach space. AIMS Math. 2021, 6, 52-65. [CrossRef]

9. Regan, D.O. Weak solutions of ordinary differeintial equation in Banach spaces. Appl. Math. Lett. 1999, 12, 101-105. [CrossRef]

10. Salem, H.A.H.; El-Sayed, A.M.A. Weak solution for fractional order integral equations in reflexive Banach spaces. Math. Slovaca 2005, 55, 169-181.

11. Salem, H.A.H.; El-Sayed, A.M.A.; Moustafa, O.L. A note on the fractional calculus in Banach spaces. Stud. Sci. Math. Hung. 2005, 42, 115-130. [CrossRef]

12. Banas, J.; Taoudi, M. Fixed points and solutions of operator equations for the weak topology in Banach algebras. Taiwan. J. Math 2014, 18, 871-893. [CrossRef]

13. El-Sayed, A.M.A.; Hashem, H.H.G. A Coupled systems of integral equations in reflexive Banach spaces. Acta Math. Sci. 2012, 32, 1-8. [CrossRef]

14. El-Sayed, A.M.A.; Hashem, H.H.G. Existence results for nonlinear quadratic integral equations of fractional order in Banach algebra. Fract. Calc. Appl. Anal. 2013, 16, 816-826. [CrossRef] 
15. Banaś, J.; Dronka, J. Integral operators of Volterra-Stieltjes type, their properties and applications. Math. Comput. Model. 2000, 32, 1321-1331. [CrossRef]

16. Banaś, J.; Sadarangani, K. Solvability of Volterra-Stieltjes operator-integral equations and their applications. Comput. Math. Appl. 2001, 41, 1535-1544. [CrossRef]

17. Banaś, J.; Mena, J.C. Some Properties of Nonlinear Volterra-Stieltjes Integral Operators. Comput. Math. Appl. 2005, 49, 1565-1573. [CrossRef]

18. Banaś, J.; O’Regan, D. Volterra-Stieltjes integral operators. Math. Comput. Model. 2005, 41, 335-344. [CrossRef]

19. El-Sayed, A.M.A.; Omar, Y.M.Y. On the Solutions of a Delay Functional Integral Equation of Volterra-Stieltjes Type. Int. J. Appl. Comput. Math 2020, 6, 8. [CrossRef]

20. Sidorov, D.N. Existence and blow-up of Kantorovich principal continuous solutions of nonlinear integral equations. Differ. Equ. 2014, 50, 1217-1224. [CrossRef]

21. Hille, E.; Phillips, R.S. Functional Analysis and Seme-Groups; American Mathematical Society: Providence, RI, USA, 1957; Volume 31

22. O'Regan, D. Fixed point theory for weakly sequentially continuous mapping. Math. Comput. Model. 1998, 27, 1-14. [CrossRef]

23. Kolmogorov, A.N.; Fomin, S.V. Introductory Real Analysis; Dover Publications Inc.: New York, NY, USA, 1975.

24. Atangana, A.; Akgül, A.; Owolabi, K.M. Analysis of fractal fractional differential equations. Alex. Eng. J. 2020, 59, 1117-1134. [CrossRef]

25. Baskonus, H.M.; Sánchez-Ruiz, L.M.; Ciancio, A. New Challenges Arising in Engineering Problems with Fractional and Integer Order. Fractal Fract. 2021, 5, 35. [CrossRef]

26. El-Sayed, A.M.A.; Gaafar, F.M. Fractional calculus and some intermediate physical processes. Appl. Math. Comput. 2003, 144, 117-126. [CrossRef]

27. Kunze, H.E.; Torre, D.L.; Mendivil, F.; Ruiz-Galán, M.; Zaki, R. Fractal-Based Methods and Inverse Problems for Differential Equations: Current State of the Art. Math. Probl. Eng. 2014, 2014, 737694. [CrossRef] 